\title{
Apelin-13 alleviates diabetic nephropathy by enhancing nitric oxide production and suppressing kidney tissue fibrosis
}

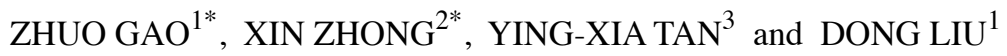 \\ ${ }^{1}$ Department of Nephrology, Air Force Medical Center, Beijing 100142; ${ }^{2}$ Department of Nephrology, \\ Longgang District Central Hospital of Shenzhen, Shenzhen, Guangdong 518116; \\ ${ }^{3}$ Institute of Transfusion Medicine, Academy of Military Medical Sciences, Beijing 100850, P.R. China
}

Received March 29, 2021; Accepted June 9, 2021

DOI: $10.3892 / \mathrm{ijmm} .2021 .5008$

\begin{abstract}
Diabetes is a serious metabolic disease, and the kidney damage induced by diabetes also seriously affects the survival of patients. Apelin is a molecule that plays a crucial role in lipid metabolism, and recent studies have revealed that apelin-13, a subtype of apelin, plays an important role in regulating blood glucose levels. However, the role of apelin-13 in diabetic nephropathy remains unclear. In the present study, a rat model of diabetic nephropathy was constructed by the injection of streptozocin (STZ). During this process, these rats were injected with apelin-13. The blood glucose, urine protein and insulin levels were determined weekly. Next, the expression of angiotensin domain type 1 receptor-associated protein (APJ), endothelial nitric oxide synthase (eNOS), E-cadherin and $\alpha$-smooth muscle actin ( $\alpha$-SMA) in the kidney tissues was determined with western blotting. Then, the endothelial cells of glomerular vessels were cultured with high glucose medium. These cells were treated with apelin-13 for $24 \mathrm{~h}$. Finally, cell viability of these cells and the expression of APJ, eNOS, E-cadherin and $\alpha$-SMA in these cells were determined with western blotting. As a result, treatment of apelin-13 induced the lower levels of blood glucose and urine protein. In addition, application of apelin-13 promoted the production of insulin and alleviated the insulin resistance. Treatment with apelin-13 promoted the expression of APJ, eNOS and E-cadherin while it suppressed the expression of $\alpha$-SMA in kidney tissues of rats and endothelial cells of glomerular vessels. Furthermore, application of apelin-13 also promoted the cell viability of these cells. In conclusion, apelin-13 relieved diabetic nephropathy by promoting the production of nitric oxide (NO) and alleviating the fibrosis of kidney tissues.
\end{abstract}

Correspondence to: Professor Zhuo Gao, Department of Nephrology, Air Force Medical Center, 30 Fucheng Road, Beijing 100142, P.R. China

E-mail: gaozhuo2007@163.com

${ }^{*}$ Contributed equally

Key words: diabetes, diabetic nephropathy, apelin-13, fibrosis, nitric oxide

\section{Introduction}

Diabetes is a metabolic disease and glucose metabolism disorder is the main clinical manifestation (1). Previous studies have indicated that, due to the popularity of high-sugar and high-fat diets and the sedentary lifestyle of individuals, the incidence of diabetes is anticipated to continue to increase in the next few years $(2,3)$. The onset of diabetes is often accompanied by damage to several organs and patients with long-term diabetes are often diagnosed with severe renal failure $(4,5)$. A previous study reported that $30 \%$ of patients with type 1 diabetes and $25 \%$ of patients with type 2 diabetes developed diabetic nephropathy (6). Furthermore, the occurrence and development of diabetic nephropathy led to an increase in the mortality of patients with diabetes. Diabetic nephropathy-induced renal podocyte and capillary damage is the main factor affecting the survival of patients (7). Therefore, developing a treatment for diabetic nephropathy is crucial for improving the survival rate of patients with diabetes.

Apelin is located at the chromosomal region Xq25-26.1 and encoded by the APLN gene (8) and is the endogenous ligand of the $\mathrm{G}$ protein-coupled receptor angiotensin domain type 1 receptor-associated protein (APJ), which is usually secreted and produced by white adipose tissue (9). Apelin and its receptor APJ are widely expressed in various tissues, including cerebellar endothelial cells, heart and blood vessels, especially in the cardiovascular system (10). Apelin was identified as an adipokine associated with lipid metabolism in a recent study (11). However, previous study has indicated that apelin plays a crucial role in regulating the energy homeostasis of the body, glucose and water metabolism as well as other biological functions (12). Apelin expression has been previously revealed to improve insulin resistance, atherosclerosis and diabetes-induced vascular damage (13). However, a different study revealed that apelin expression induced the occurrence and development of diabetic retinopathy (14). Therefore, the role of apelin in the occurrence and development of diabetes-related complications remains unclear.

Furthermore, apelin is present as different subtypes (apelin-36, apelin-17 and apelin-13) in the body through the shear of the endoplasmic reticulum. It was revealed that the shorter the peptide chain of apelin, the stronger its biological activity (15). A previous study indicated that apelin improved 
insulin sensitivity and induced lower blood sugar levels by reducing chronic inflammation in patients with type 2 diabetes, thereby enhancing glucose and lipid metabolism (16). However, the effect of apelin on diabetic nephropathy is currently controversial. A previous study has reported that the levels of apelin/APJ in the serum of patients with diabetic nephropathy are elevated, promoting the occurrence and development of diabetic nephropathy (17). On the other hand, a different study revealed that apelin-13 could inhibit the morphological changes of renal tubular epithelial cells in the high-glucose environment, thereby inhibiting the occurrence and development of the epithelial-mesenchymal transition (EMT) process in glomerular cells, and ultimately delaying the occurrence of diabetic nephropathy (18). Therefore, the effect of apelin on diabetes-induced renal complications remains unclear.

In the present study, the role of apelin-13 in the process of diabetic nephropathy was further investigated by constructing a rat model of diabetic nephropathy.

\section{Materials and methods}

Animal assays. A total of 30 Sprague Dawley male rats (6-8 weeks old; 200-230 g) were obtained from the Beijing Vital River Laboratory Animal Technology Co., Ltd. and housed in a temperature-controlled $\left(25^{\circ} \mathrm{C}\right)$ and specific pathogen-free room with constant humidity (40-50\%) in a 12-h light/dark cycle and received food and water ad libitum for 1 week. These rats were then equally divided into six groups (Control, Model, Ap-E40, Ap-E400, Ap-L40 and Ap-L400). The rats in the control group were treated with nothing. The rats of the other groups were fed with a high-sugar and high-fat diet (Thermo Fisher Scientific, Inc.) and received an intravenous injection of streptozocin (STZ; $40 \mathrm{mg} / \mathrm{kg}$; product no. S0130; Merck $\mathrm{KGaA}$ ) every week. After 3 weeks, the Ap-E40 and Ap-E400 rats were treated with apelin-13 (40 and $400 \mathrm{pmol} / \mathrm{kg}$, respectively; product no. A6469; Merck KGaA). A total of 6 weeks after the STZ injection, the rats of the Ap-L40 and Ap-L400 groups were treated with apelin-13 (40 and $400 \mathrm{pmol} / \mathrm{kg}$, respectively). After eight STZ injections, the rats were euthanized with sodium pentobarbital (160 mg/kg, i.p.). Following euthanasia, the serum and organs were collected. The weight of the rats was detected using electronic scales every week, from 1 week prior to the STZ injection and until euthanasia. Blood glucose levels were detected every week using a blood glucose meter (Thermo Fisher Scientific, Inc.). Urine protein levels were also detected using a BCA assay kit (cat. no. P0012S; Beyotime Institute of Biotechnology) every week. The fasting serum insulin was also detected every week using a commercial kit (cat. no. RAB0904; Merck KGaA) according to the manufacturer's instructions. The index of insulin resistance was calculated using the following formula: (Insulin levels in the blood $\mathrm{x}$ blood glucose levels)/22.5. All the experiments were performed according to the Guide for the Care and Use of Laboratory Animals 8th edition (19) and approved (approval no. 2020-79-PJ01) by the Ethics Committee of the Air Force Medical Center (Beijing, China).

Detection of blood urea nitrogen (BUN) and serum creatinine (Scr). The levels of BUN (cat. no. m1076479) and Scr (cat. no. ml059158) in the blood were determined using commercial kits (Shanghai Enzyme-linked Biotechnology Co., Ltd.) after 3 and 8 weeks of STZ injection, respectively. All steps of this assay were performed according to the manufacturers' instructions.

Detection of nitric oxide (NO), angiotensin II (Ang II) and endothelin-1 (ET-1). The levels of NO, Ang II and ET-1 in the serum and nephridial tissues were detected using NO kit (cat. no. S0021; Beyotime Institute of Biotechnology), Ang II kit (product no. RAB0010; Merck KGaA) and ET-1 kit (cat. no. E-EL-R1458c; Elabscience Biotechnology, Inc.). All steps of the assays were performed according to the manufacturers' instructions.

Hematoxylin and eosin $(H \& E)$ staining. Following euthanasia, kidney tissues were collected and washed with pre-cooled phosphate buffer saline (PBS). The kidney was then separated and fixed with $4 \%$ paraformaldehyde overnight at room temperature. The paraffin-embedded kidney tissues were cut into $5-\mu \mathrm{m}$ sections. Next, these sections were dehydrated in 70 and $90 \%$ ethanol solution for 10 min each. Finally, H\&E staining at room temperature for $3 \mathrm{~min}$ was performed to detect pathological changes. All steps of this assay were performed according to the manufacturer's instructions of the H\&E staining kit (cat. no. C0105S; Beyotime Institute of Biotechnology).

Immunohistochemistry. Immunohistochemistry was carried out using the standard peroxidase/DAB method and hematoxylin counterstaining according to the manufacturer's instructions. After being blocked with $5 \%$ bovine serum albumin (BSA; Beyotime Institute of Biotechnology) for $20 \mathrm{~min}$ at room temperature, sections were incubated with anti-APJ primary antibody (cat. no. PA5-114830; Thermo Fisher Scientific, Inc.) overnight at $4^{\circ} \mathrm{C}$. After PBS washing, sections were incubated with goat anti-rabbit horseradish peroxidase-conjugated secondary antibody (product code ab6721; $1: 1,000$; Abcam) at $4^{\circ} \mathrm{C}$ for $50 \mathrm{~min}$, followed by the PBS washing. Then, sections were treated with DAB solution and counterstained with hematoxylin at room temperature for $5 \mathrm{~min}$. Finally, the images were obtained using a light microscope (Olympus Corporation).

Cell culture and treatment. A human renal glomerular microvascular endothelial cell line (hRGEC) was obtained from the BioVector NTCC, Inc. and cultured in RPMI-1640 medium (Thermo Fisher Scientific, Inc.), supplemented with $10 \%$ fetal bovine serum (FBS) in humidified air at $37^{\circ} \mathrm{C}$ with $5 \% \mathrm{CO}_{2}$. These cells were divided into the control, model, Ap-L (Apelin-low) and Ap-H (Apelin-high) groups. Model, Ap-L and Ap-H group cells were cultured in high-glucose (25 mmol/1; Cytiva) RPMI-1640 medium and control group cells were cultured in RPMI-1640 medium with normal glucose content $(5.5 \mathrm{mmol} / \mathrm{l})$. Next, Ap-L group cells were treated with low levels of apelin-13 $(0.1 \mu \mathrm{mol} / \mathrm{l})$ and Ap-H group cells were treated with high levels of apelin-13 (1 $\mu \mathrm{mol} / \mathrm{l})$.

Tube formation experiment. Tube formation in hRGEC cells was determined using a commercial kit (product no. K905-50; AmyJet Scientific, Inc.). All images were obtained using a 
light microscope (magnification, $\mathrm{x} 4$; Olympus Corporation). All steps of this assay were performed according to the manufacturer's instructions.

Cell Counting Kit-8 (CCK-8) assay. hRGEC cells from various groups were plated into three 96 -well plates $\left(2.5 \times 10^{4} /\right.$ well). After 24,48 and $72 \mathrm{~h}$ of incubation at $37^{\circ} \mathrm{C}, 10 \mu \mathrm{lCCK}-8$ reagent (Dojindo Molecular Technologies, Inc.) was diluted with the culture medium and added to the 96-well plates. Next, these cells were placed in an incubator for $1 \mathrm{~h}$. Finally, the absorbance of these cells was detected using a spectrophotometer (Thermo Fisher Scientific, Inc.) at a wavelength of $450 \mathrm{~nm}$.

Western blotting. Total proteins were collected from nephridial tissue and hRGEC cells using RIPA lysis buffer (Santa Cruz Biotechnology, Inc.). Protein concentration was determined using a BCA assay kit. Next, equal amounts of protein $(40 \mu \mathrm{g})$ were separated via $10 \%$ sodium dodecyl sulfate-polyacrylamide gel electrophoresis (SDS-PAGE) (Beyotime Institute of Biotechnology) and transferred to a polyvinylidene fluoride (PVDF) membrane (EMD Millipore), which was blocked with $5 \%$ bovine serum albumin (BSA) (Beyotime Institute of Biotechnology) at $37^{\circ} \mathrm{C}$ for $1.5 \mathrm{~h}$. The membranes were then incubated with the following primary antibodies at $4^{\circ} \mathrm{C}$ overnight: Ang II type 1 receptor (AT1R; 1:1,000; product code ab124734; Abcam), endothelial NO synthase (eNOS; 1:1,000; product ocde ab5589; Abcam), APJ (1:1,000; cat. no. PA5-114830; Thermo Fisher Scientific, Inc.), transforming growth factor $\beta$ receptor (TGBR; 1:1,000; product code ab97459; Abcam), E-cadherin (1:1,000; product code ab212059; Abcam), $\alpha$-smooth muscle actin ( $\alpha$-SMA; 1:1,000; product no. 19245; Cell Signaling Technology, Inc.) and GAPDH (1:2,500; product code ab9485; Abcam). Following the primary antibody incubation, the membranes were washed with PBS-Tween-20 (0.1\%) and incubated with HRP-conjugated antibody (1:2,000; product code ab6721; Abcam) for $2 \mathrm{~h}$ at room temperature (7) Finally, the immunoreactive signals were assessed using a Pierce ${ }^{\mathrm{TM}}$ ECL Western Blotting Substrate kit (Thermo Fisher Scientific, Inc.) according to the manufacturer's instructions. The relative intensities of the immunoblots were measured with a Versa Doc $^{\mathrm{TM}}$ MP 5000 molecular imager and Quantity One software (version 4.6) (both from Bio-Rad Laboratories, Inc.).

Statistical analysis. Statistical analysis was performed using GraphPad Prism 7.0 (GraphPad Software, Inc.). Unpaired Student's t-tests were used to compare two groups. Comparisons among different groups were assessed using one-way ANOVA followed by Tukey's post-hoc test. All data are presented as the mean \pm standard deviation (SD) and all experiments were repeated three times. $\mathrm{P}<0.05$ was considered to indicate a statistically significant difference.

\section{Results}

Apelin-13 decreases the weight and blood glucose levels, and increases insulin levels of diabetic nephropathy rats at 8 weeks. The weight and blood glucose levels of rats were detected weekly during the process of raising them. The results (Fig. 1A and $\mathrm{B}$ ) revealed that the weight and blood glucose levels of the rats in the Ap-E40 and Ap-E400 groups were not significantly altered after the injection of apelin-13 at 3 weeks. The same parameters in the rats of the Ap-L40 and Ap-L400 groups were decreased after the apelin-13 injection at 8 weeks compared with that at 3 weeks. The levels of insulin in the blood were also decreased after the apelin-13 injection at 8 weeks compared with that at 3 weeks (Fig. 1C).

Apelin-13 therapy improves renal function of diabetic nephropathy rats. The increased BUN and Scr levels indicated the occurrence and development of renal injury (20). BUN and Scr levels were revealed to be higher after the apelin-13 injection at 8 weeks compared with that at 3 weeks (Fig. 2A). Similarly, the urinary protein content and extent of pathological injury of renal tissues also revealed the same trends (Fig. 2B and C).

Apelin-13 alleviates diabetic nephropathy by modulating the production of NO, AngII and ET-1. The expression of APJ was detected and the results (Fig. 3A) revealed that the expression of APJ in the renal tissues of rats was decreased after the apelin-13 injection at 8 weeks compared with that at 3 weeks. NO production could relieve the diabetic nephropathy-induced vascular damage in the renal tissue (21). Therefore, the production of NO, Ang II and ET-1 was detected in the renal tissues. The results revealed that the levels of NO were decreased after the injection of apelin-13 at 8 weeks compared with that at 3 weeks. However, the levels of Ang II and ET-1 were increased after the injection of apelin-13 at 8 weeks compared with that at 3 weeks (Fig. 3B and C). Next, the expression of eNOS and AT1R was detected by western blotting. As revealed in Fig. 4A, the expression of AT1R was suppressed after the injection of apelin-13 at 3 and 8 weeks compared with the model group, respectively. The levels of eNOS were increased after the injection of apelin-13 at 3 and 8 weeks compared with the model group.

Apelin-13 alleviates diabetic nephropathy by modulating the expression of AT1R, endothelial nitric eNOS and renal fibrosis-related proteins in APJ pathway. The expression of APJ was increased and that of TGBR was decreased after the injection of apelin-13 at 3 and 8 weeks compared with the model group. The expression levels of the fibrosis-related proteins, E-cadherin and $\alpha$-SMA, were detected, and the results revealed that the expression of E-cadherin was enhanced and the levels of $\alpha$-SMA were decreased after the injection of apelin-13 at 3 and 8 weeks compared with the model group (Fig. 4B).

Apelin-13 promotes proliferation and tube formation, and modulates the expression of renal fibrosis-related proteins in the APJ pathway in short-term high glucose-induced renal vascular endothelial cells. hRGEC cells were cultured in high-glucose medium for 24,48 and $72 \mathrm{~h}$. Next, a CCK-8 assay was performed to detect cell viability. The results (Fig. 5A) revealed that treatment with apelin-13 promoted cell viability. The application of apelin-13 also promoted lumen formation (Fig. 5B). At $24 \mathrm{~h}$ of apelin-13 treatment, it was revealed that the expression levels of APJ, eNOS and E-cadherin were increased and the levels of TGBR, AT1R and $\alpha$-SMA were decreased compared with the model group (Fig. 5C). 

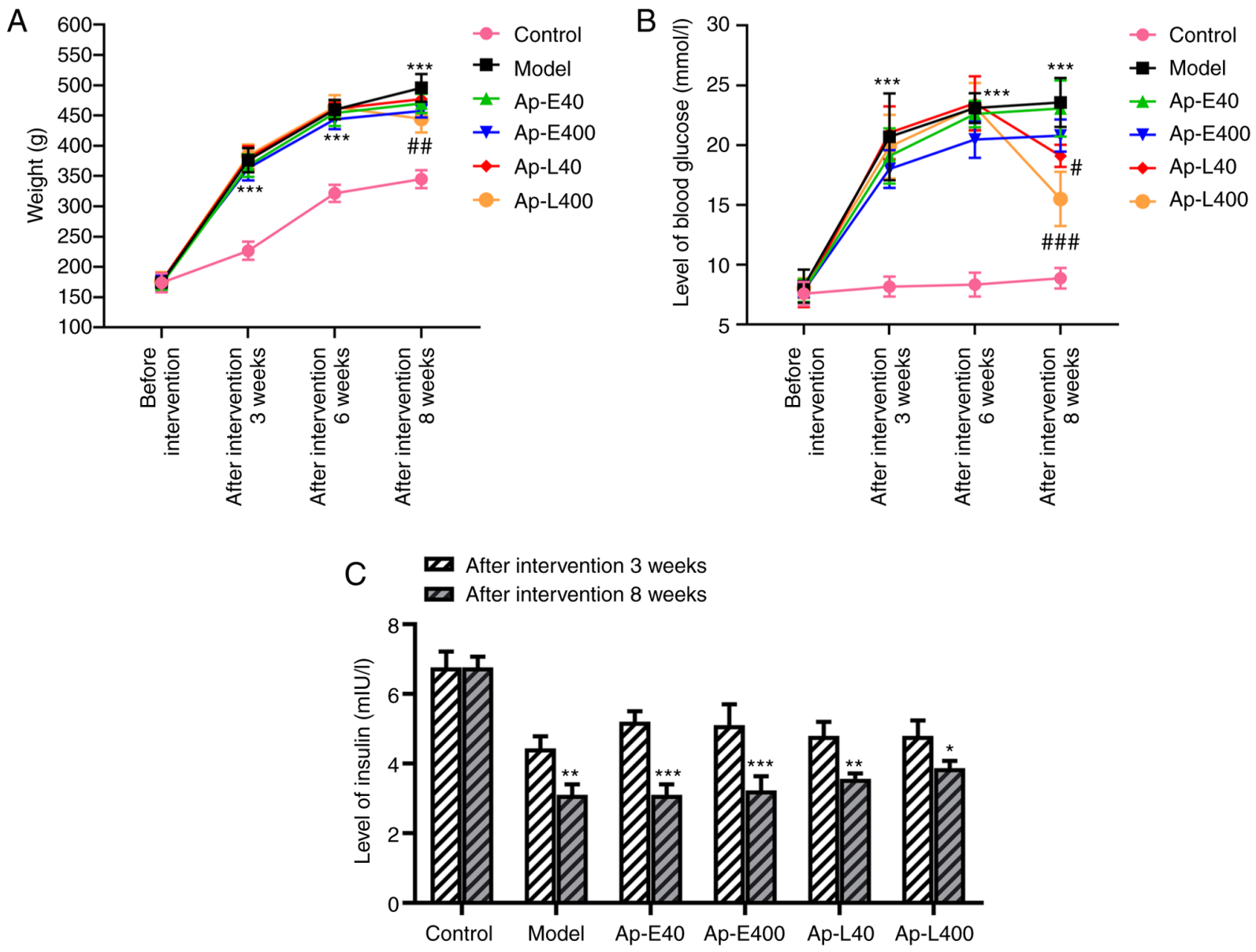

Figure 1. Apelin-13 relieves the insulin resistance of rats. (A) The weight of mice was detected weekly. (B) Blood glucose levels were detected weekly. ${ }^{* * * *} \mathrm{P}<0.001$ vs. the Control group. ${ }^{\#} \mathrm{P}<0.05,{ }^{\# \#} \mathrm{P}<0.01$ and ${ }^{\# \# \#} \mathrm{P}<0.001$ vs. the Model group. (C) Levels of insulin were detected. ${ }^{*} \mathrm{P}<0.05,{ }^{* * *} \mathrm{P}<0.01$ and ${ }^{* * * *} \mathrm{P}<0.001$ vs. the After intervention $3 \mathrm{w}$ group.

Apelin-13 suppresses proliferation and tube formation, and modulates the expression of renal fibrosis-related proteins in the APJ pathway in long-term high glucose-induced renal vascular endothelial cells. Cells were treated with high-glucose medium for 72 and $96 \mathrm{~h}$, and interference with apelin-13 began from $72 \mathrm{~h}$. After $24 \mathrm{~h}$, the CCK-8 assay results revealed that the application of apelin-13 supressed cell viability compared with the model group (Fig. 6A). Lumen formation was inhibited following treatment with apelin-13 (Fig. 6B). In addition, treatment with apelin-13 also promoted the expression of APJ, eNOS and E-cadherin compared with the model group. The application of apelin-13 also suppressed the expression of TGBR, AT1R and $\alpha$-SMA (Fig. 6C) compared with the model group in a dose-dependent manner.

\section{Discussion}

Diabetes is a metabolic disease and glucose metabolism disorder is its main clinical manifestation. In recent years, the incidence of this disease has been steadily increasing (22). Diabetes leads to the damage and dysfunction of several organs. Diabetes-induced kidney damage is one of the most prominent causes of mortality in patients with diabetes (23). The main clinical manifestations of diabetic nephropathy have been revealed to be proteinuria and persistent decline in renal function (24). In addition, the occurrence and development of diabetic nephropathy have been revealed to promote the development of cardiovascular disease (25). Therefore, the need to develop a new therapy to suppress the damage caused by diabetic nephropathy in patients is urgent.

Apelin is a fat factor in adipose tissues. A recent study reported that apelin could relieve the clinical manifestations of patients with diabetes by regulating blood glucose levels (26). Another recent study reported that apelin-36 could inhibit insulin secretion induced by high-concentration glucose $(16.7 \mathrm{mmol} / \mathrm{l})$, but had no effect on insulin secretion induced by low-concentration glucose $(2.8 \mathrm{mmol} / \mathrm{l})(27)$. In the bodies of obese and insulin-resistant mice, apelin could relieve insulin resistance and restore the use of glucose in the body (28). However, in the field of diabetic nephropathy, a recent study reported that the levels of apelin and its receptor APJ in the serum of patients with diabetic nephropathy were increased, and higher levels of apelin and APJ promoted blood vessel formation and induced the proliferation of glomerular capillaries, thereby promoting the occurrence and development of diabetic nephropathy (29). However, a different study also indicated that apelin-13 could inhibit the transformation of renal tubular epithelial cells in a high-glucose environment, thereby inhibiting the occurrence and development of the EMT process in glomerular cells, ultimately delaying the occurrence of diabetic nephropathy (30). In the present study, it was demonstrated that the use of apelin-13 decreased the levels of 
A

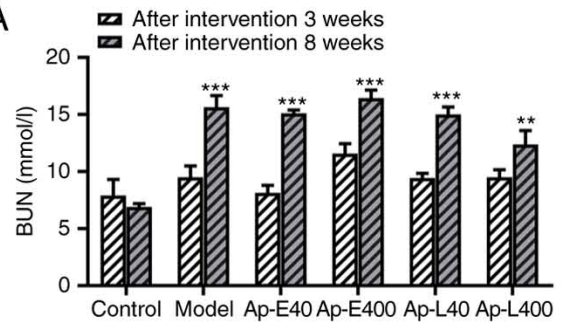

C
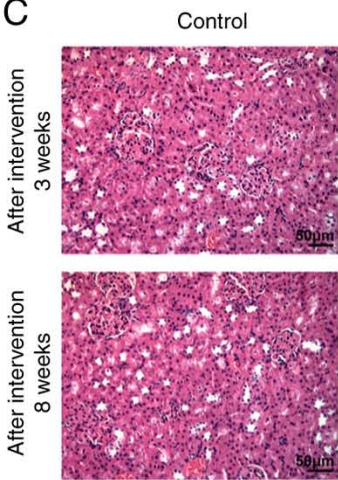

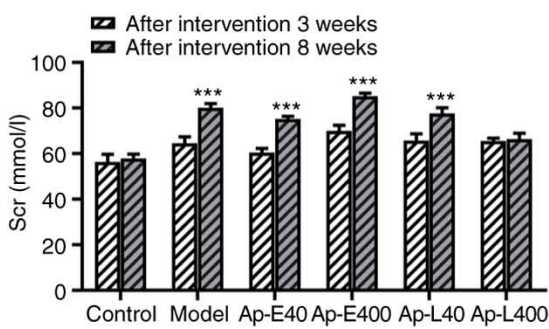

B After intervention 3 weeks

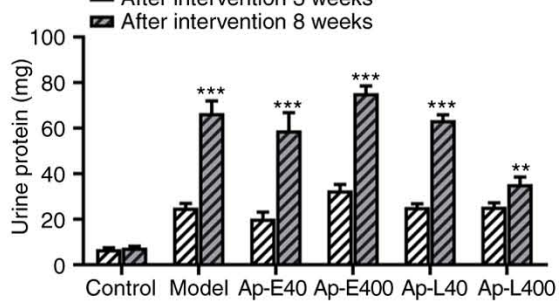

Ap- $\mathrm{L} 40$

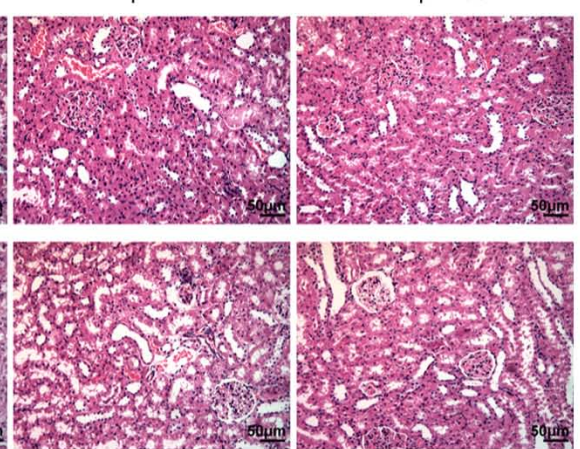

Ap-E400

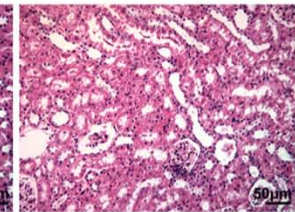

Figure 2. Treatment of apelin-13 alleviates the diabetes-induced kidney damage of rats. (A) The levels of blood urea nitrogen and serum creatinine were detected. (B) The urinary protein content was detected weekly. (C) The injury of kidney tissues was detected with the hematoxylin and eosin staining (magnification, $\mathrm{x} 200) .{ }^{* *} \mathrm{P}<0.01$ and ${ }^{* * *} \mathrm{P}<0.001$ vs. the After intervention $3 \mathrm{w}$ group. BUN, blood urea nitrogen; Scr, serum creatinine.

A
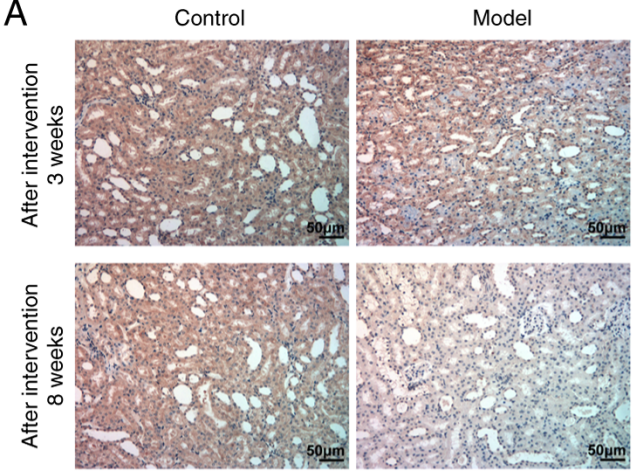

B

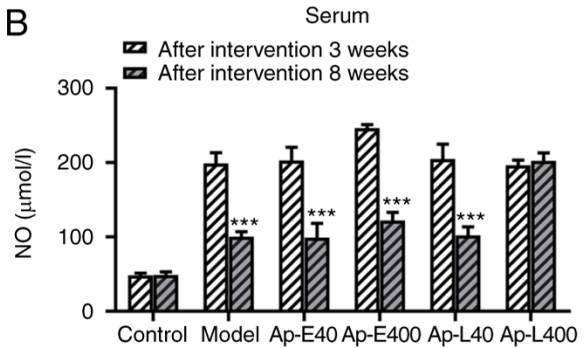

C

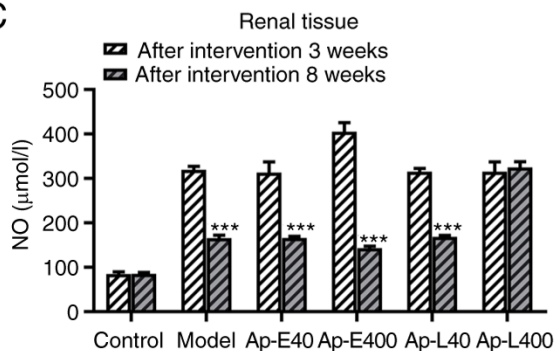

Ap-E40

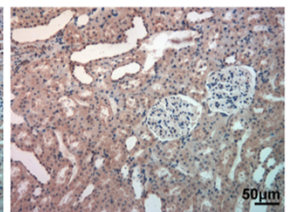

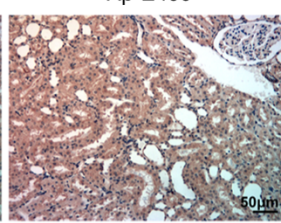

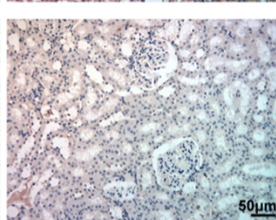

Serum

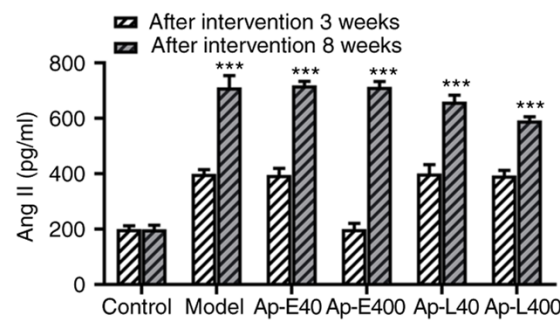

Renal tissue

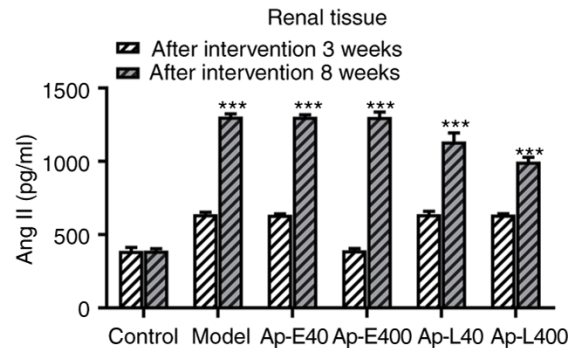

Ap-L40

Ap-L400
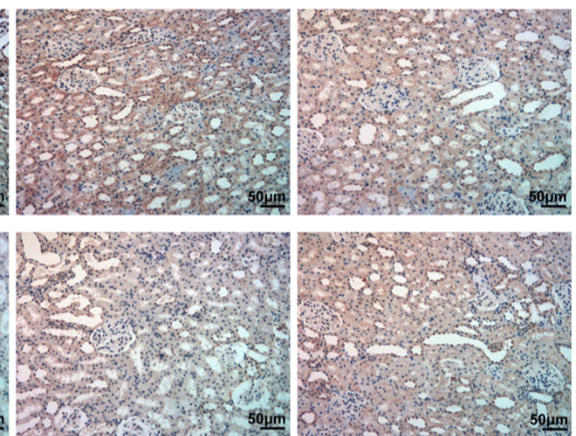

Serum

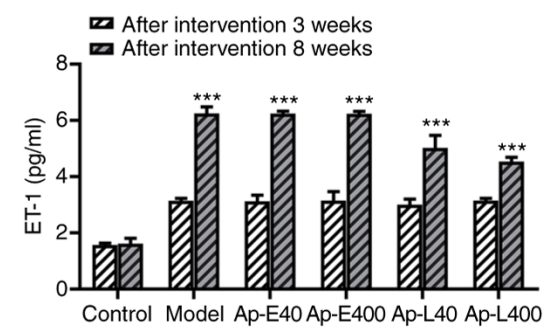

Renal tissue

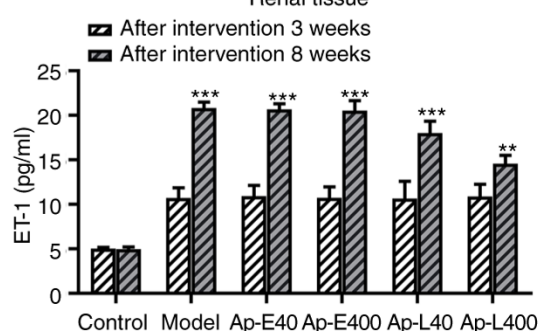

Figure 3. Apelin-13 activates the expression of APJ. (A) The expression of APJ in the kidney tissues was detected with the immunohistochemical assays (magnification, x200). (B) The production of NO was detected with the commercial kits. (C) Levels of Ang II and ET-1 were determined with commercial kits. ${ }^{* *} \mathrm{P}<0.01$ and ${ }^{* * * *} \mathrm{P}<0.001$ vs. the After intervention $3 \mathrm{w}$ group. APJ, angiotensin domain type 1 receptor-associated proteins; NO, nitric oxide; Ang II, angiotensin II; ET-1, endothelin-1. 
A
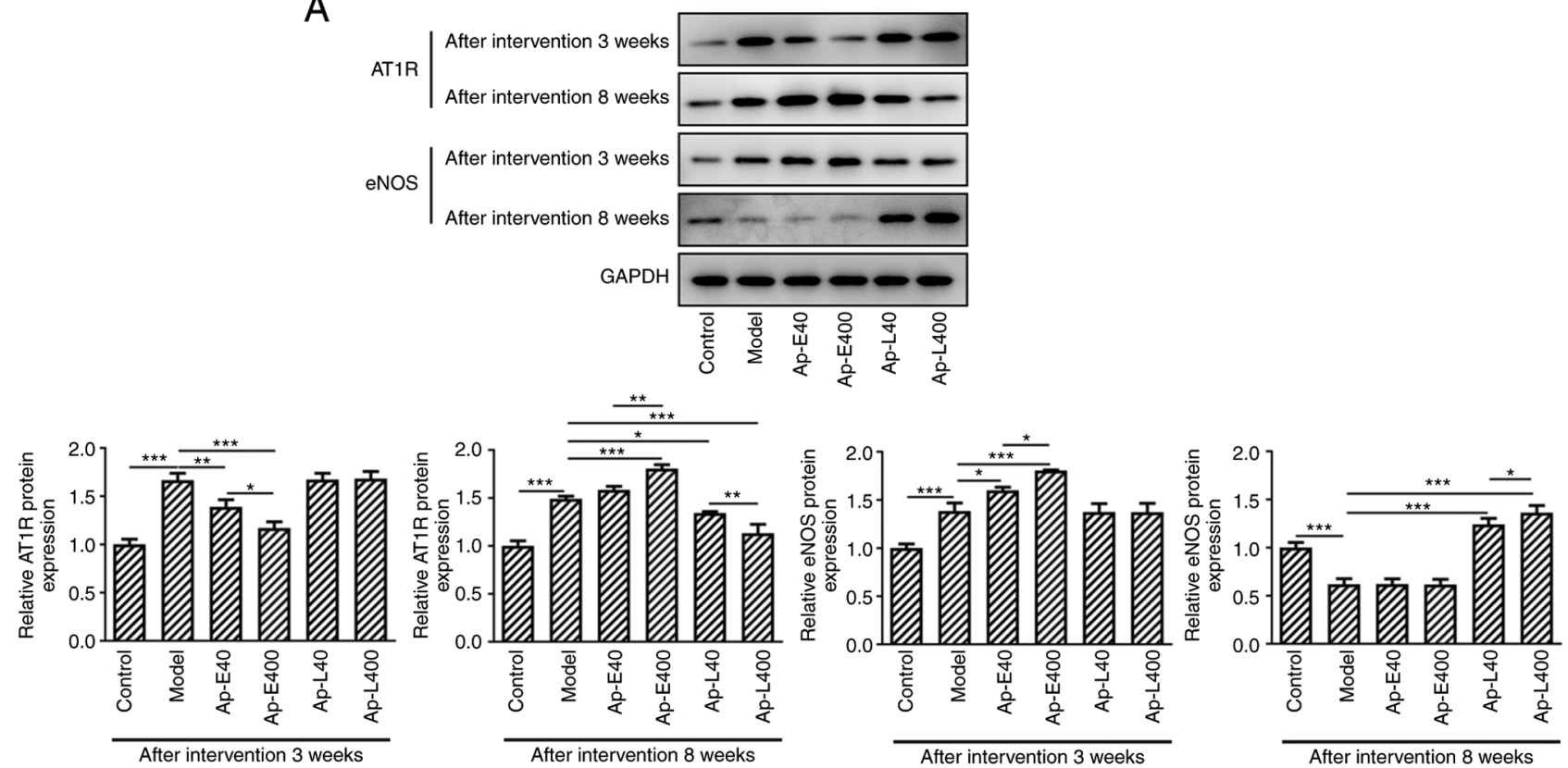

B
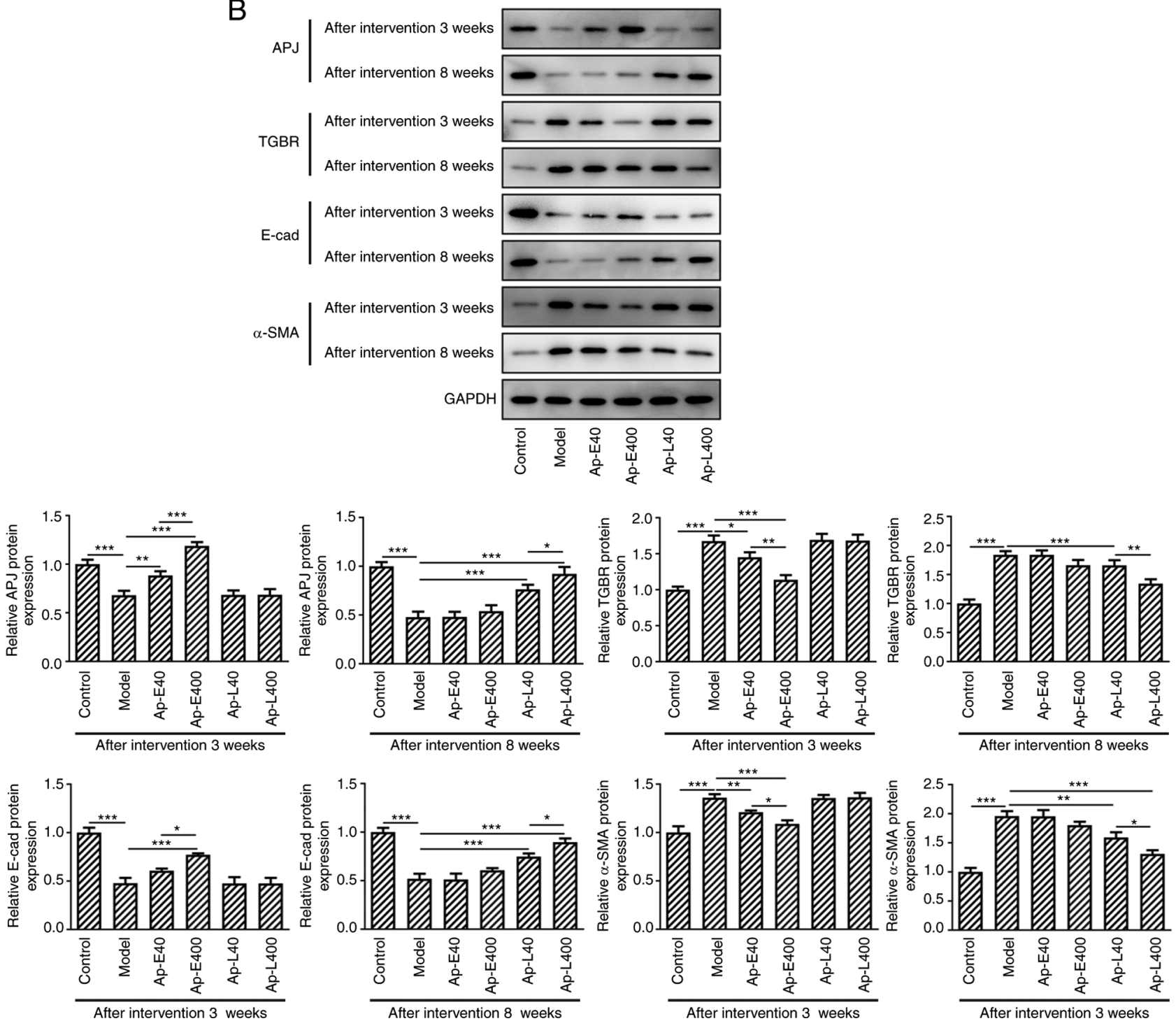

Figure 4. Application of apelin-13 promotes the expression of eNOS. (A) The expression of AT1R and eNOS was detected with the western blotting. (B) The levels of APJ, TGBR, E-cadherin and $\alpha$-SMA in kidney tissues of rats were detected with western blotting. ${ }^{*} \mathrm{P}<0.05,{ }^{* *} \mathrm{P}<0.01$ and ${ }^{* * * *} \mathrm{P}<0.001$. eNOS, endothelial nitric oxide synthase; AT1R, Ang II type 1 receptor; APJ, angiotensin domain type 1 receptor-associated proteins; TGBR, transforming growth factor $\beta$ receptor; $\alpha$-SMA, $\alpha$-smooth muscle actin. 


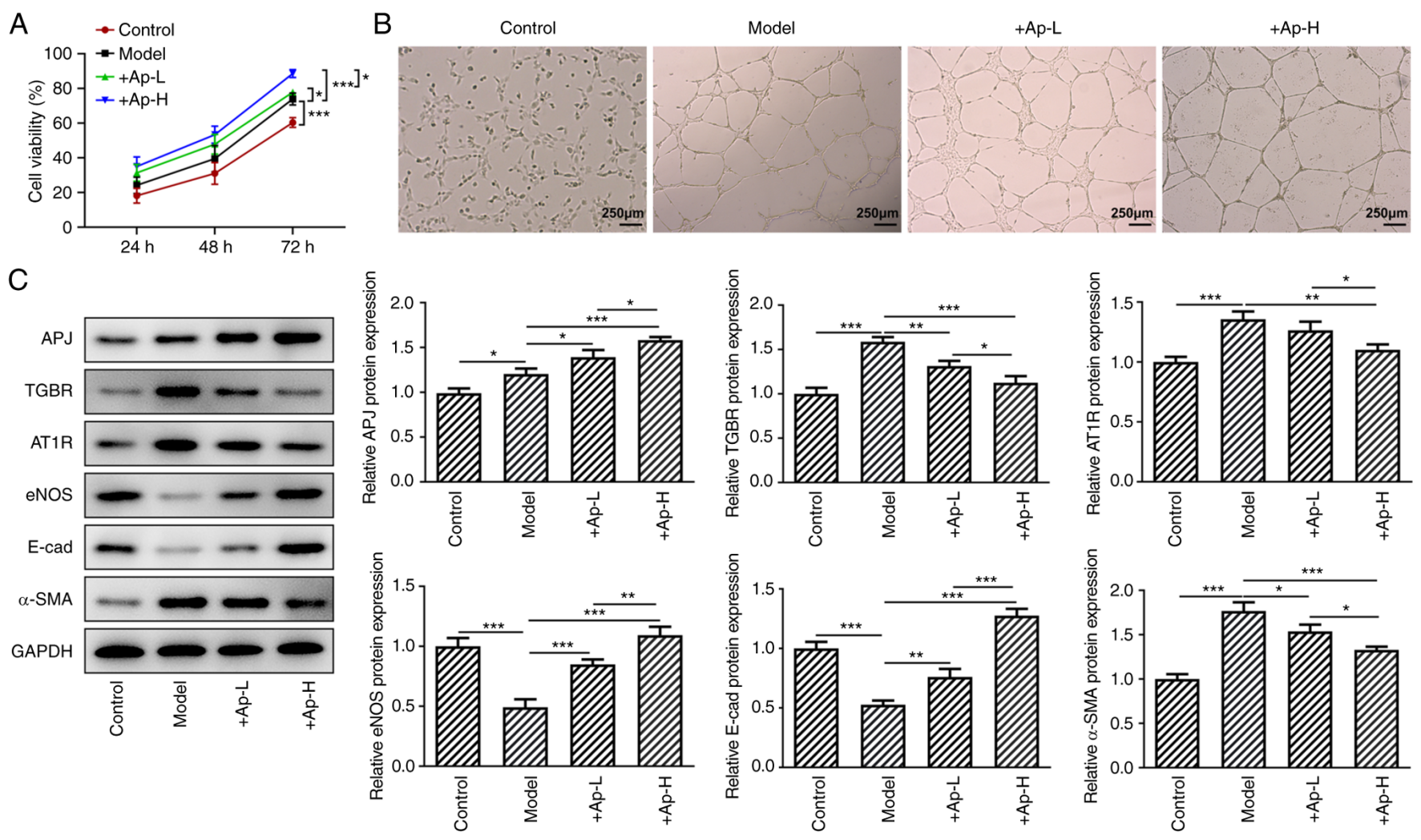

Figure 5. Apelin-13 alleviates the higher glucose-induced injury of endothelial cells of glomerular vessels. (A) Cell viability was detected with the Cell Counting Kit-8 assay. (B) hRGECs were detected with the lumen formation experiments (magnification, x4). (C) The expression of AT1R, eNOS, APJ, TGBR, E-cadherin and $\alpha$-SMA in these cells was determined with the western blotting. ${ }^{*} \mathrm{P}<0.05,{ }^{* * *} \mathrm{P}<0.01$ and ${ }^{* * * *} \mathrm{P}<0.001$. AT1R, Ang II type 1 receptor; eNOS, endothelial nitric oxide synthase; APJ, angiotensin domain type 1 receptor-associated proteins; TGBR, transforming growth factor $\beta$ receptor; $\alpha$-SMA, $\alpha$-smooth muscle actin.

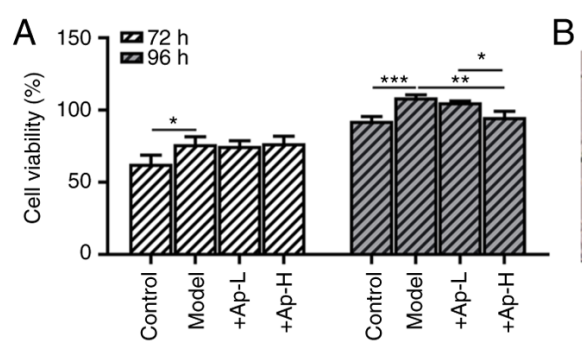

C
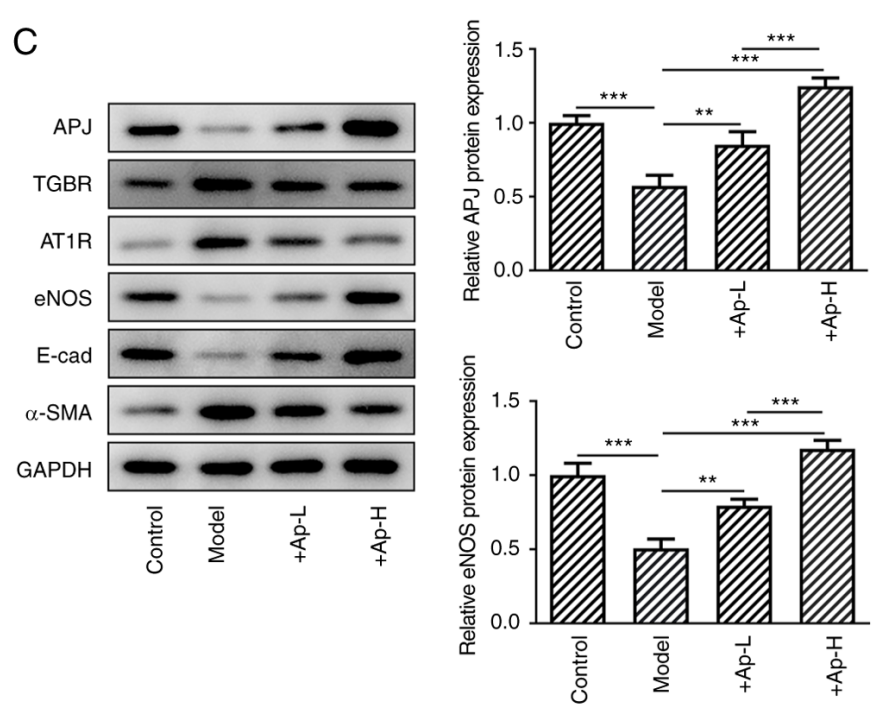

Control
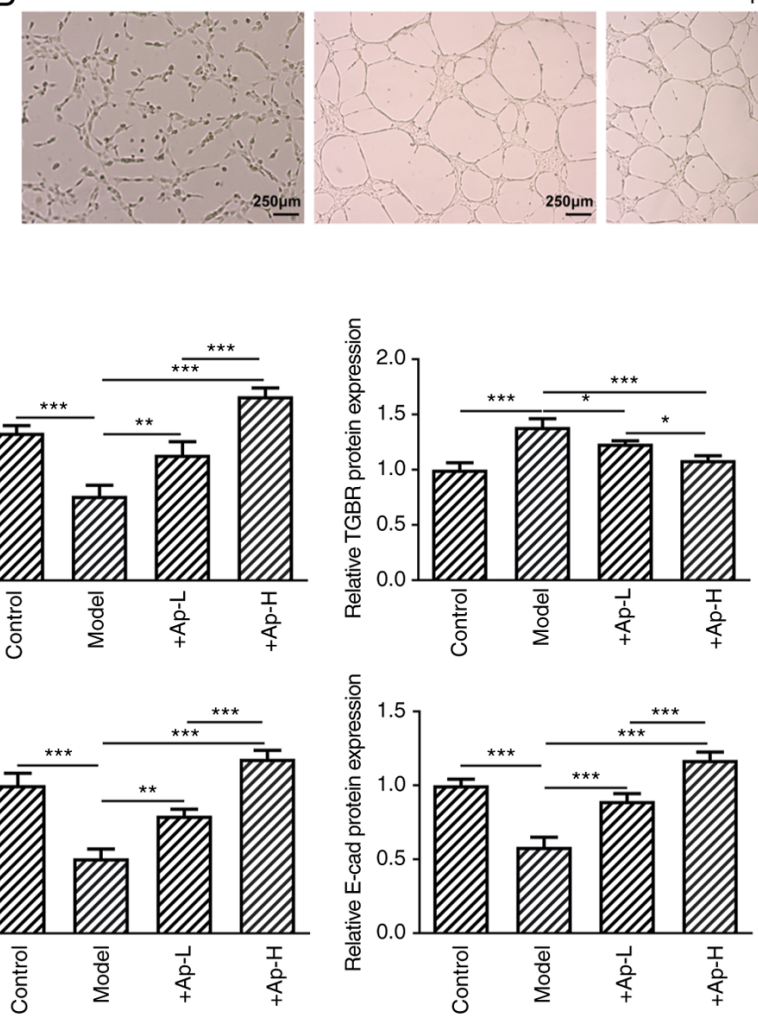

$+\mathrm{Ap}-\mathrm{L}$
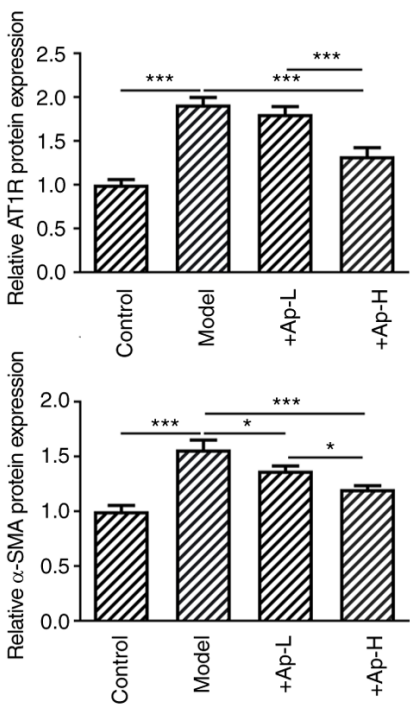

Figure 6. Apelin-13 relieves the injury of endothelial cells of glomerular vessels by suppressing fibrosis. (A) Cell viability of endothelial cells of glomerular vessels was determined with Cell Counting Kit-8 assay. (B) hRGECs were detected with the lumen formation experiments (magnification, $\mathrm{x} 4$ ). (C) The levels of AT1R, eNOS, APJ, TGBR, E-cadherin and $\alpha$-SMA in these cells were determined with western blotting. $\mathrm{P}<0.05,{ }^{* *} \mathrm{P}<0.01$ and ${ }^{* * * *} \mathrm{P}<0.001$. AT1R, Ang II type 1 receptor; eNOS, endothelial nitric oxide synthase; APJ, angiotensin domain type 1 receptor-associated proteins; TGBR, transforming growth factor $\beta$ receptor; $\alpha$-SMA, $\alpha$-smooth muscle actin. 
blood glucose and relieved insulin resistance in these rats, as well as reduced the urinary protein content. Apelin-13 treatment also alleviated the considerable glucose-induced damage in the endothelial cells of glomerular vessels. Collectively, these results indicated that the use of apelin-13 relieved the manifestations of diabetic nephropathy.

A previous study revealed that apelin could reduce blood glucose content by regulating the expression of eNOS and activating the Akt pathway (31). The expression of apelin could also produce NO by activating APJ, therefore relieving the clinical manifestations of diabetic nephropathy (32). APJ is the receptor of apelin-13, and apelin-13 could exert its biological effects by binding to the APJ (33). In the present study, the production of NO and expression of eNOS and APJ were also detected. It was revealed that the expression levels of eNOS and APJ were enhanced in renal tissues and endothelial cells of glomerular vessels following treatment with apelin-13. NO production was also enhanced in the renal tissues of rats following treatment with apelin-13. These results indicated that apelin-13 promoted the expression of eNOS by activating APJ. Therefore, higher levels of eNOS enhanced NO production, ultimately promoting vascular permeability and renal filtration.

Recent studies have indicated that renal fibrosis is a characteristic of diabetic nephropathy $(34,35)$. The results of the present study revealed that the expression of E-cadherin was enhanced in cells and renal tissues following treatment with apelin-13, while that of $\alpha$-SMA was decreased. Apelin-13 treatment was also revealed to alleviate diabetic nephropathy by suppressing the fibrosis of renal tissues and endothelial cells of glomerular vessels.

In conclusion, the present study reported the effects of apelin-13 on the development of diabetic nephropathy. The results of the present study revealed that apelin-13 alleviated diabetic nephropathy by promoting the production of NO and relieving fibrosis in renal tissues. However, the present study also has a limitation. Oxidative stress and inflammation play important roles in the development of diabetic nephropathy (36-40). Whether apelin-13 improved diabetic nephropathy by reducing inflammation and oxidative stress warrants investigation in our future study.

\section{Acknowledgements}

Not applicable.

\section{Funding}

No funding was received.

\section{Availability of data and materials}

The datasets generated during and/or analyzed during the current study are available from the corresponding author on reasonable request.

\section{Authors' contributions}

ZG conceived the study and revised the manuscript. ZG and XZ performed the experiments and were responsible for drafting manuscript. All authors (ZG, XZ, YXT and DL) performed the data analysis and organized the figures. ZG, XZ and YXT confirm the authenticity of all the raw data. All authors read and approved the final manuscript.

\section{Ethics approval and consent to participate}

All the experiments were performed according to the Guide for the Care and Use of Laboratory Animals 8th edition and approved (approval no. 2020-79-PJ01) by the Ethics Committee of the Air Force Medical Center (Beijing, China).

\section{Patient consent for publication}

Not applicable.

\section{Competing interests}

The authors declare that they have no competing interests.

\section{References}

1. Yu SY, Dong B, Fang ZF, Hu XQ, Tang L and Zhou SH: Knockdown of lncRNA AK139328 alleviates myocardial ischaemia/reperfusion injury in diabetic mice via modulating miR-204-3p and inhibiting autophagy. J Cell Mol Med 22: 4886-4898, 2018.

2. Rowley WR and Bezold C: Creating public awareness: State 2025 diabetes forecasts. Popul Health Manag 15: 194-200, 2012.

3. Rowley WR, Bezold C, Arikan Y, Byrne E and Krohe S: Diabetes 2030: Insights from yesterday, today, and future trends. Popul Health Manag 20: 6-12, 2017.

4. American Diabetes Association: Standards of medical care in diabetes-2014. Diabetes Care 37 (Suppl 1): S5-S13, 2014.

5. Ahlqvist E, van Zuydam NR, Groop LC and McCarthy MI: The genetics of diabetic complications. Nat Rev Nephrol 11: 277-287, 2015.

6. Navarro-González JF, Jarque A, Muros M, Mora C and García J: Tumor necrosis factor-alpha as a therapeutic target for diabetic nephropathy. Cytokine Growth Factor Rev 20: 165-173, 2009.

7. Alvarez ML and Distefano JK: The role of non-coding RNAs in diabetic nephropathy: Potential applications as biomarkers for disease development and progression. Diabetes Res Clin Pract 99: 1-11, 2013.

8. Del Ry S, Cabiati M, Raucci S, Simioniuc A, Caselli C, Prescimone T and Giannessi D: Sequencing and cardiac expression of Apelin in Sus Scrofa. Pharmacol Res 60: 314-319, 2009.

9. Fasshauer $\mathrm{M}$ and Blüher M: Adipokines in health and disease. Trends Pharmacol Sci 36: 461-470, 2015.

10. Lv D, Li H and Chen L: Apelin and APJ, a novel critical factor and therapeutic target for atherosclerosis. Acta Biochim Biophys Sin (Shanghai) 45: 527-533, 2013.

11. Wysocka MB, Pietraszek-Gremplewicz K and Nowak D: The role of apelin in cardiovascular diseases, obesity and cancer. Front Physiol 9: 557, 2018.

12. Sentinelli F, Capoccia D, Bertoccini L, Barchetta I, Incani M, Coccia F, Manconi E, Lenzi A, Cossu E, Leonetti F, et al: Search for genetic variant in the apelin gene by resequencing and association study in European subjects. Genet Test Mol Biomarkers 20: 98-102, 2016

13. Onalan E, Yakar B, Barım AO and Gursu MF: Serum apelin and resistin levels in patients with impaired fasting glucose, impaired glucose tolerance, type 2 diabetes, and metabolic syndrome. Endokrynol Pol 71: 319-324, 2020.

14. Adki KM and Kulkarni YA: Potential biomarkers in diabetic retinopathy. Curr Diabetes Rev 16: 971-983, 2020.

15. Wu R, Zhu Z and Zhou D: VEGF, apelin and HO-1 in diabetic patients with retinopathy: A correlation analysis. BMC Ophthalmol 20: 326, 2020.

16. Yasir M, Senthilkumar GP, Jayashree K, Ramesh Babu K, Vadivelan $\mathrm{M}$ and Palanivel C: Association of serum omentin-1, apelin and chemerin concentrations with the presence and severity of diabetic retinopathy in type 2 diabetes mellitus patients. Arch Physiol Biochem: Nov 5, 2019 (Epub ahead of print). 
17. Sabouri M, Norouzi J, Zarei Y, Sangani MH and Hooshmand Moghadam B: Comparing high-intensity interval training (HIIT) and continuous training on Apelin, APJ, NO, and cardiotrophin-1 in cardiac tissue of diabetic rats. J Diabetes Res 2020: 1472514, 2020.

18. Dawood AF, Sabry MM, Estaphan SA, Mohamed EA, Younes SF, Rashed LA and Elzainy AW: Cross-talk between apelin and vasopressin in response to different osmotic stimuli in type 2 diabetic rats. J Biol Regul Homeost Agents 32: 1117-1127, 2018.

19. National Research Council (US) Committee for the Update of the Guide for the Care and Use of Laboratory Animals: Guide for the Care and Use of Laboratory Animals. 8th edition. National Academies Press (US), Washington, DC, 2011.

20. Chen Y, Lin L, Tao X, Song Y, Cui J and Wan J: The role of podocyte damage in the etiology of ischemia-reperfusion acute kidney injury and post-injury fibrosis. BMC Nephrol 20: 106, 2019.

21. Xue M, Shi Y, Pang A, Men L, Hu Y, Zhou P, Long G, Tian X, Wang R, Zhao Y, et al: Corin plays a protective role via upregulating MAPK and downregulating eNOS in diabetic nephropathy endothelial dysfunction. FASEB J 34: 95-106, 2020

22. Rossi L and Gesualdo L: Diabetic nephropathy and cardiovascular risk. G Ital Nefrol 34 (Suppl 69): S104-S118, 2017 (In Italian).

23. Van Krieken R and Krepinsky JC: Caveolin-1 in the pathogenesis of diabetic nephropathy: Potential therapeutic target? Curr Diab Rep 17: 19, 2017

24. Saran R, Robinson B, Abbott KC, Agodoa LYC, Bhave N, Bragg-Gresham J, Balkrishnan R, Dietrich X, Eckard A, Eggers PW, et al: US renal data system 2017 annual data report: Epidemiology of kidney disease in the United States. Am J Kidney Dis 71 (3 Suppl 1): A7, 2018.

25. Khan NU, Lin J, Liu X, Li H, Lu W, Zhong Z, Zhang H, Waqas M and Shen L: Insights into predicting diabetic nephropathy using urinary biomarkers. Biochim Biophys Acta Proteins Proteom 1868: 140475, 2020.

26. Castan-Laurell I, El Boustany R, Pereira O, Potier L, Marre M, Fumeron F, Valet P, Gourdy P, Velho G and Roussel R: Plasma Apelin and risk of type 2 diabetes in a cohort from the community. Diabetes Care 43: e15-e16, 2020.

27. Guo YY, Li T, Liu H, Tang L, Li YC, Hu HT, Su YF, Lin Y, Wang YY, Li C, et al: Circulating levels of Elabela and Apelin in the second and third trimesters of pregnancies with gestational diabetes mellitus. Gynecol Endocrinol 36: 890-894, 2020.

28. Tanday N, Irwin N, Moffett RC, Flatt PR and O'Harte FPM: Beneficial actions of a long-acting apelin analogue in diabetes are related to positive effects on islet cell turnover and transdifferentiation. Diabetes Obes Metab 22: 2468-2478, 2020.

29. Bae JH, Kwak SE, Lee JH, Yangjie Z and Song W: Does exercise-induced apelin affect sarcopenia? A systematic review and meta-analysis. Hormones (Athens) 18: 383-393, 2019.
30. Sabry MM, Mahmoud MM, Shoukry HS, Rashed L, Kamar SS and Ahmed MM: Interactive effects of apelin, renin-angiotensin system and nitric oxide in treatment of obesity-induced type 2 diabetes mellitus in male albino rats. Arch Physiol Biochem 125: 244-254, 2019

31. Ji B, Shang L, Wang C, Wan L, Cheng B and Chen J: Roles for heterodimerization of APJ and B2R in promoting cell proliferation via ERK1/2-eNOS signaling pathway. Cell Signal 73: 109671, 2020

32. Nagib AM, El-Diasty A, El Husseny MA, El-Gamal EM, Abbas MH, Refaie AF and Foudas MA: Apelin and new-onset diabetes after transplant in living kidney allograft recipients. Exp Clin Transplant 13: 319-323, 2015.

33. Huang Z, Luo X, Liu M and Chen L: Function and regulation of apelin/APJ system in digestive physiology and pathology. J Cell Physiol 234: 7796-7810, 2019.

34. Wakisaka M, Kamouchi M and Kitazono T: Lessons from the trials for the desirable effects of sodium glucose Co-transporter 2 inhibitors on diabetic cardiovascular events and renal dysfunction. Int J Mol Sci 20: 5668, 2019.

35. Li A, Peng R, Sun Y, Liu H, Peng $\mathrm{H}$ and Zhang Z: LincRNA 1700020I14Rik alleviates cell proliferation and fibrosis in diabetic nephropathy via miR-34a-5p/Sirt1/HIF-1 $\alpha$ signaling. Cell Death Dis 9: 461, 2018

36. Zhou X, Ma L, Habibi J, Whaley-Connell A, Hayden MR, Tilmon RD, Brown AN, Kim JA, Demarco VG and Sowers JR: Nebivolol improves diastolic dysfunction and myocardial remodeling through reductions in oxidative stress in the Zucker obese rat. Hypertension 55: 880-888, 2010.

37. Wada $J$ and Makino $H$ : Innate immunity in diabetes and diabetic nephropathy. Nat Rev Nephrol 12: 13-26, 2016.

38. Niewczas MA, Pavkov ME, Skupien J, Smiles A, Md Dom ZI, Wilson JM, Park J, Nair V, Schlafly A, Saulnier PJ, et al: A signature of circulating inflammatory proteins and development of end-stage renal disease in diabetes. Nat Med 25: 805-813, 2019.

39. Mou Z, Feng Z, Xu Z, Zhuang F, Zheng X, Li X, Qian J and Liang G: Schisandrin B alleviates diabetic nephropathy through suppressing excessive inflammation and oxidative stress. Biochem Biophys Res Commun 508: 243-249, 2019.

40. Al Hroob AM, Abukhalil MH, Alghonmeen RD and Mahmoud AM: Ginger alleviates hyperglycemia-induced oxidative stress, inflammation and apoptosis and protects rats against diabetic nephropathy. Biomed Pharmacother 106: 381-389, 2018. 